\title{
Discovering and Reconciling Semantic Conflicts: A Data Mining Perspective
}

\author{
Hongjun Lu Weiguo Fan Cheng Hian Goh \\ Department of Information Systems \& Computer Science \\ National University of Singapore \\ \{luhj,fanweigu,gohch\}@iscs.nus.sg
}

\author{
Stuart E. Madnick \\ Sloan School of Mgt \\ MIT \\ smadnick@mit.edu
}

\author{
David W. Cheung \\ Dept of Computer Science \\ Hong Kong University \\ dcheung@cs.hku.hk
}

\begin{abstract}
Current approaches to semantic interoperability require human intervention in detecting potential conflicts and in defining how those conflicts may be resolved. This is a major impedance to achieving "logical connectivity", especially when the number of disparate sources is large. In this paper, we demonstrate that the detection and reconciliation of semantic conflicts can be automated using tools and techniques developed by the data mining community. We describe a process for discovering such rules that represent the relationships among semanticaly related attributes and illustrate the effectiveness of our approach with examples.
\end{abstract}

\section{Keywords}

Database Integration, Data Mining, Regression Analysis, Semantic Conflicts

\section{INTRODUCTION}

A variety of online information sources and receivers (i.e., users and applications) has emerged at an unprecedented rate in the last few years, contributed in large part by the exponential growth of the Internet as well as advances in telecommunications technology. Nonetheless, this increased physical connectivity (the ability to exchange bits and bytes) does not necessarily lead to logical connectivity (the ability to exchange information meaningfully). This problem is sometimes referred to as the need for semantic interoperability (Sheth \& Larson 1990) among autonomous and heterogeneous systems.

Traditional approaches to achieving semantic interoperability can be iden- 
tified as either tightly-coupled or loosely-coupled (Scheuermann, Yu, Elmagarmid, Garcia-Molina, Manola, McLeod, Rosenthal \& Templeton Dec 1990). In tightly-coupled systems, semantic conflicts are identified and reconciled a priori in one or more shared schema, against which all user queries are formulated. This is typically accomplished by the system integrator or DBA who is responsible for the integration project. In a loosely-coupled system, conflict detection and resolution is the responsibility of users, who must construct queries that take into account potential conflicts and identify operations needed to circumvent them. In general, conflict detection and reconciliation is known to be a difficult and tedious process since the semantics of data are usually present implicitly and often ambiguous. This problem poses even greater difficulty when the number of sources increases exponentially and when semantics of data in underlying sources changes over time.

It should be clear from the preceding discussion that automating the detection and reconciliation process will be an important step forward in achieving the intelligent integration of information. In this paper, we show how data mining techniques can be gainfully employed to this end. Our strategy consists of the application of statistical techniques to overlapping subsets of data present in disparate sources, through which rules for data conversion may be extracted.

The remainder of this paper is organized as follows. Section 2 presents a scenario in which integrated access to heterogeneous information sources is required. We observed that whenever information content in disparate sources overlap, we will have an opportunity for engaging in the automatic discovery of conflicts and their resolution. Section 3 presents a statistical approach for conflict detection and resolution and introduces the supporting techniques. Section 4 presents details of three experiments which are used in illustrating and validating the approach taken. Finally, Section 5 summarizes our findings and describes the issues which need to be resolved in the near future.

\section{MOTIVATIONAL EXAMPLE}

Consider the scenario depicted in Figure 1. Both databases keep information about hotels, for which some are identical in both databases. For the purpose of this discussion, we assume that key-values used for identifying individual hotels are common to the two databases: e.g., the hotel with $h_{-}$code 1001 in both LOCALDB and REMOTEDB refers to the same hotel.

Observe however that although both databases have an attribute rate, different values are reported by the two databases even when the same real world entity is referenced. This anomaly arises from the fact that the two databases have a different interpretation of rate: in the case of REMOTEDB, hotel rates are reported without taking applicable state taxes into consideration; on the other hand, hotel rates in LOCALDB are reported as charges "after-tax". 
$L O C A L D B$ :

hotel (ㄴ_code, location, breakfast, facilities, rate);

\begin{tabular}{lcccc}
\hline \multicolumn{5}{c}{ hotel } \\
\hline h_code & location & breakfast & facility & rate \\
\hline 1001 & 1 & 0 & 0 & 53 \\
1002 & 2 & 1 & 1 & 88 \\
1003 & 1 & 1 & 2 & 106 \\
2001 & 5 & 1 & 2 & 200 \\
\hline
\end{tabular}

\section{REMOTEDB :}

hotel (h_code, state, breakfast, facilities, rate);

tax_rate (state, tax_rate);

\begin{tabular}{lcccc}
\hline \multicolumn{5}{c}{ hotel } \\
\hline$h$ code & state & meal & bath & rate \\
\hline 1001 & 1 & 0 & 0 & 50 \\
\hline 1002 & 2 & 1 & 1 & 80 \\
\hline 1003 & 1 & 1 & 2 & 100 \\
\hline 3001 & 1 & 1 & 0 & 120 \\
\hline
\end{tabular}

\begin{tabular}{cc}
\hline \multicolumn{2}{c}{ tax_rate } \\
\hline state & rate \\
\hline 1 & 6 \\
\hline 2 & 10 \\
\hline 3 & 15 \\
\hline 4 & 18 \\
\hline
\end{tabular}

Figure 1 Two example databases.

Intuitively, we say that a semantic conflict exists between REMOTEDB and LOCALDB over attribute rate.

The meaningful exchange of information across different systems requires all semantic conflicts to be resolved. For instance, it would not be meaningful to write a query which compares hotel rates in REMOTEDB to those in LOCALDB without reconciling the conflict over attribute rate in the two databases. In the current example, this conflict can be resolved by adding the tax-amount to the hotel rates reported in REMOTEDB. Notice that the computation of tax-value requires the multiplication of the hotel rates in REMOTEDB with a corresponding tax rate corresponding to the state in which the hotel resides. We refer to the operation underlying the data transformation as a conversion function. Despite the critical role it plays in enabling semantic interoperability, the identification of appropriate conversion functions is a difficult task given that the assumptions underlying data representation are not always made explicit.

In general, conversion functions may take on various different forms. For example, it may be a lookup table which maps one string representation to another (e.g., "IBM" to "International Business Machine"), or it may be any arbitrary "black-box" function (e.g., a dvips program which transforms a 
.dvi file to a .ps file). For our purpose in this paper, we consider only conversion functions which are arithmetic expressions. Although this represents only a subset of all possible conversion functions, arithmetic conversions are commonly encountered in practice in a variety of different domains and are therefore important in their own right.

The remaining discussion will be presented using the relational data model without any loss of generality. For simplicity, we will use Horn clauses for representing conversion functions. For the example described earlier, we will write

L.hotel(hcode, state, breakfast, facilities, roomrate2) $\leftarrow$

R.tax_rate(state, tax_rate),

R.hotel(hcode, state, breakfast, facilities, roomrate1), roomrate $2=(1+0.01 *$ tax_rate $) *$ roomrate 1.

In the rest of this paper, we will demonstrate how conversion functions such as the one above can be discovered through the use of tools and techniques developed by the data mining community.

\section{A STATISTICAL APPROACH FOR CONFLICT DETECTION AND RESOLUTION}

Discovery of conversion functions proceeds in three steps. In Step 1 (semantic relevance analysis), we use the techniques of correlation analysis to isolate attributes which are potentially semantically-related. In Step 2, we may rely on human intervention to identify attributes in distinct databases which are to be treated as synonyms (even though these may embody semantic conflicts). Finally, in Step 3 (Quantitative Relationship Analysis), we apply regression analysis to generate the relevant conversion functions that allow values in one databases to be transformed to values that are meaningful in a different context.

In the remainder of this section, we furnish the details of the statistical techniques employed in our approach.

\subsection{Semantic Relevance Analysis}

Given two sets of attributes from two databases, the objective of semantic relevance analysis is to find those attributes which either represent the same real world properties, or are derivable from each other. Such analysis can be conducted at two levels: at the metadata level or at the data level. In this study, we only discuss the second: i.e., we will attempt to isolate a subset of the attributes which are semantically-related by analyzing the data set, as 
opposed to relying on a thesaurus that tries to make sense of the attribute names (e.g., as in suggesting that attributes revenue and income are related).

If we view the attributes as variables, the problem we have on hand is similar to the problem of statistical correlation analysis. Given two variables, $X$ and $Y$ and their measurements $\left(x_{i}, y_{i}\right) ; i=1,2, \ldots, n$, we shall attempt to measure the strength of association between the two variables by means of a correlation coefficient $r$. The value of $r$ is between -1 and +1 with $r=0$ indicating the absence of any linear association between $X$ and $Y$. Intuitively, larger values of $\boldsymbol{r}$ indicate a stronger association between the variables being examined. A value of $r$ equal to -1 or 1 implies a perfect linear relation. While correlation analysis can reveal the strength of linear association, it is based on an assumption that $X$ and $Y$ are the only two variables under the study. If there are more than two variables, other variables may have some effects on the association between $X$ and $Y$. Partial Correlation Analysis (PCA), is a technique that provides us with a single measure of linear association between two variables while adjusting for the linear effects of one or more additional variables. Properly used, partial correlation analysis can uncover spurious relationships, identify intervening variables, and detect hidden relationships that are present in a data set.

In our study, we use PCA to analyze the semantic relevance among attributes. Given two databases $D_{1}$ and $D_{2}$, we can treat the records in relations as measurements. Let $R_{1}\left(A_{1}^{1}, A_{2}^{1}, \ldots, A_{m}^{1}\right)$ with primary key $A_{1}^{1}$ be a relation from $D_{1}$, and $R_{2}\left(A_{1}^{2}, A_{2}^{2}, \ldots, A_{n}^{2}\right)$ with primary key $A_{1}^{2}$ be a relation from $D_{2}$. We also assume that two records $r_{1}$ of $R_{1}$ and $r_{2}$ of $R_{2}$ refer to the same "real world" entity if $r_{1} \cdot A_{1}^{1}=r_{2} . A_{1}^{2}$. Therefore, we can form measurements using records with the same key value from the two relations. Partial correlation analysis can be then applied to the data sets with $(m+n-2)$ variables (where $m$ and $n$ are the number of attributes in the two relations under investigation). The details of the various steps will be illustrated in the next section.

Note that, correlation and semantic relevance are two different concepts. A person's height and weight may be highly correlated, but it is obvious that height and weight are different in their semantics. However, since semantic conflicts come from the difference in representation scheme and such representational difference is uniformly applied to each entity, high correlation should exist among the values of semantically related attributes. Correlation analysis can at least isolate the attributes which are likely to be related to one another for further analysis. In our approach exemplified in this paper, this step provides a preliminary clustering of semantically-related attributes which are then presented to respective user groups, who must now define the attributes which are to be treated as synonyms. Synonyms nonetheless may continue to embody semantic conflicts. When these conflicts can be resolved through arithmetic transformations, the underlying quantitative relationships can be uncovered automatically as described in the next section. 


\subsection{Quantitative Relationship Analysis}

The discovery of quantitative laws from data sets is a classic machine learning problem: to this date, various systems have been reported in the literature. Examples of these systems include BACON (Langley, Simon, G.Bradshaw \& Żytkow 1987), ABACUS (Falkenhainer \& Michalski 1986) and COPER (Kokar 1986). BACON, being one of the earliest systems, has made some interesting re-discoveries in physics and chemistry (Langley et al. 1987). ABACUS improved upon BACON and is capable of discovering multiple laws (Falkenhainer \& Michalski 1986). In the case of COPER (Kokar 1986), physical laws which can be modeled by a polynomial function can also be uncovered. A recently reported system, KEPLER (Wu \& Wang 1991), is an interactive system which is able to check data correctness and discover multiple equations. Another system, FORTY-NINER, borrows solutions from the early systems and adjusts them to the specific needs of database exploration (Żytkow \& Baker 1991).

One of the basic techniques for discovering quantitative laws is a statistical technique known as regression analysis. Thus, if we view the database attributes as variables, the relationships we are looking for among the attributes are nothing more than regression functions. For example, the equation roomrate2 $=(1+0.01 *$ tax_rate $) *$ roomrate 1 can be viewed as a regression function, where roomrate2 is the response (dependent) variable, tax_rate and roomrate1 are predictor (independent) variables. In general, there are two steps in using regression analysis. First step is to define a model, which is a prototype of the function to be discovered. For example, a linear model of $p$ independent variables can be expressed as follows:

$Y=\beta_{0}+\beta_{1} X_{1}+\beta_{2} X_{2}+\ldots+\beta_{p} X_{p}+\mu$

where $\beta_{0}, \beta_{1}, \ldots, \beta_{p}$ are model parameters, or regression coefficients, and $\mu$ is an unknown random variable that measures the departure of $Y$ from exact dependence on the $p$ predictor variables.

With a defined model, the second step in regression analysis is to estimate the model parameters $\beta_{1}, \ldots, \beta_{p}$. Various techniques have been developed for the task. The essence of these techniques is to fit all data points to the regression function by determining the coefficients. The goodness of the discovered function with respect to the given data is usually measured by the coefficient of determination, or $R^{2}$. The value $R^{2}$ ranges between 0 and 1 . A value of 1 implies perfect fit of all data points with the function.

In our experiments reported in the next section, regression analysis is used primarily to discover the quantitative relationships among semanticallyrelated attributes. One essential difference between the problem of discovering conversion functions and the classic problem of discovering quantitative laws is the nature of the data. Data from experimental results from which quanti- 
tative laws are to be discovered contains various errors, such as measurement errors. On the other hand, semantic conflicts we are dealt with are due to different representations which are uniformly applied to all entities, the discovered function that resolves the conflicts should apply to all records. If the database contains no erroneous entries, the discovered function should perfectly cover the data set. In other words, functions discovered using regression analysis should have $R^{2}=1$.

In some instances, the $R^{2}$ value for a regression function discovered may not be 1 . For example, this may be the case when the cluster does not contain all attributes required to solve the conflicts; in which case, no perfect quantitative relationship can be discovered at all. Moreover, it is also possible that the conflicts cannot be reconciled using a single function, but are reconcilable using a suitable collection of different functions. To accommodate for this possibility, we extend the procedure by partitioning the data set (whenever a suitable regression function cannot be found) and attempting to identify an appropriate regression function for each of the partition taken one at a time. This partitioning can be done in a number of different ways. For our purpose, we use the simple heuristic of partitioning the data set using categorical attributes present in a relation. The rationale is, if multiple functions exist for different partitions of data, data records in the same partition must have some common property, which is most likely reflected by values assumed by some attributes within the databases being investigated.

\section{AUTOMATIC DISCOVERY OF CONVERSION FUNCTIONS}

To provide a concrete illustration of this approach proposed in the preceding section, we describe below three experiments. The first two are based on the scenario introduced in the motivational example; the last presents a simplified version of a real example which we have encountered.

\subsection{Experiment One}

We shall consider the databases REMOTEDB and LOCALDB as shown earlier. Since hotel and tax_rate are in the same database, we assume that they do not have any conflicts on the attribute state. To simplify the ensuing discussion, we shall assume that these two tables are "joined" to yield the relation shown in Figure 2; the attribute rate in LOCALDB and REMOTEDB are prefixed with $L$ and $R$ respectively to avoid any ambiguity. The attributes in this relation will be used as inputs to Step 1 of our procedure and serve as the basis for identifying attributes which are semantically-related. For simplicity, we shall also ignore attributes meal and bath in the joined database since they have the same value as breakfast and facility.

To allow us to demonstrate the validity of data mining techniques for the 


\begin{tabular}{lcccccccc}
\hline h_code & location & breakfast & facility & L.rate & meal & bath & R.rate & taxrate \\
\hline 1001 & 1 & 0 & 0 & 53 & 0 & 0 & 50 & 6 \\
1002 & 2 & 1 & 1 & 88 & 1 & 1 & 80 & 10 \\
1003 & 1 & 1 & 2 & 106 & 1 & 2 & 100 & 6 \\
\hline
\end{tabular}

Figure 2 The relation obtained by joining relations in REMOTEDB and LOCALDB.

discovery of semantic knowledge in the form of conversion functions, we created the synthetic databases corresponding to LOCALDB and REMOTEDB. This is accomplished by generating the values of the attributes identified earlier using a uniform distribution, according to Table 1. Values of L.roomrate are generated using the following formula:

L.roomrate $=(1+0.01 *$ taxrate $) *$ R.roomrate

The number of tuples in the dataset is 1000 .

Table 1 Domain values used in materializing the synthetic databases.

\begin{tabular}{llllll}
\hline attribute & state & taxrate & facility & breakfast & R.roomrate \\
\hline values & $0-40$ & $1-18$ & $\{0,1,2\}$ & $\{0,1\}$ & $20-500$ \\
\hline
\end{tabular}

We now present detailed descriptions of the three steps corresponding to our strategy.

\section{Identifying semantically related attributes}

To identify attributes which may be semantically-related, a partial correlation analysis is performed on the dataset. Table 2 lists the result.

It can be seen that if 0.1 is chosen as a threshold, L.roomrate, taxrate, and $R$.roomrate will be considered to be correlated. To verify this, we further perform a PCA analysis while controlling for the other three attributes, i.e. state, breakfast, and facility. Table 3 shows the result of the analysis:

We can see from Table 3 that L.roomrate and $R$.roomrate indeed have a very strong linear relationship. But the relationship between L.roomrate and R.taxrate is not so obvious (0.1447). To further determine whether L.roomrate and $R$.taxrate are correlated, we make another partial correlation analysis between them by controlling $R$.roomrate. The result is shown in Table 4. As can be seen from Table 4 that L.roomrate does correlate 
Table 2 Zero-Order Partials for Experiment 1.

\begin{tabular}{lrrrrrr} 
& L.roomrate & breakfast & facility & taxrate & R.roomrate & state \\
\hline L.roomrate & 1.0000 & & & & & \\
breakfast & .0396 & 1.0000 & & & & \\
facility & .0320 & .0567 & 1.0000 & & & \\
taxrate & .1447 & .0067 & .0927 & 1.0000 & & \\
R.roomrate & .9958 & .0386 & .0241 & .0626 & 1.0000 & \\
state & .0040 & .0124 & -.0584 & -.0530 & .0071 & 1.0000 \\
\hline
\end{tabular}

Table 3 PCA results when controlling for state, breakfast and facility.

\begin{tabular}{lrrr} 
& L.roomrate & taxrate & R.roomrate \\
\hline L.roomrate & 1.0000 & & \\
taxrate & .1429 & 1.0000 & \\
R.roomrate & .9958 & .0611 & 1.0000 \\
\hline
\end{tabular}

highly with taxrate. We therefore conclude that attribute L.roomrate is highly related to two attributes, taxrate and R.roomrate, in the remote database.

One interesting observation from the above result is that the association between state(location) and L.roomrate or R.roomrate is very low. This can be explained as follows: For the same state, different hotels have different room rates, which make the linear association between state and roomrate not very strong. The same reason also applies to the correlation between state and taxrate. Since different states may have the same tax rate, the correlation between them is very low, either.

\section{Defining the synonyms}

Table 4 PCA results when controlling for R.roomrate.

\begin{tabular}{lrr} 
& L.roomrate & taxrate \\
\hline L.roomrate & 1.0000 & \\
taxrate & .8975 & 1.0000 \\
\hline
\end{tabular}


From a user or application perspective, certain attributes present in different databases may be treated as synonyms. Thus the rate attribute in both LOCALDB and REMOTEDB are treated as synonyms (having the same meaning). Notice that these two attributes may or may not be identical syntactic tokens: i.e., having the same attribute name (although they happen to be the same in this instance). Furthermore, two attributes may be viewed as synonyms by one user and are yet semantically distinct from another user's viewpoint. Hence, a knowledgeable user may understand the semantic disparity between hotel rates reported in REMOTEDB and LOCALDB and therefore choose to treat the two attributes as different. For this reason, it is mandatory for synonyms are to be defined by users depending on their understanding. This however does not mean that users will have to peruse through the thousands of attributes which are present in disparate databases. Clearly, the attributes which have been identified in Step 1 above serve as a useful starting point whereby potential synonyms can be identified.

Notice that there are no right or wrong synonyms. If two attributes are identified as synonyms, they will be used as input to the next step in the discovery process in which an attempt is made to identify the relationship between the two. Two attributes which are semantically-related in some way may however not be labeled as synonyms. For instance, the attribute rate could have been treated as distinct in REMOTEDB and LOCALDB. This however will not pose any problem as long as the user does not attempt to compare them under the assumption that they are referring to the same thing.

\section{Discovering the relationship}

The final step in our process is to find the quantitative relationship among attributes which are identified as synonyms. For the example at hand, we need to find the relationship among L.roomrate, taxrate and R.roomrate, assuming that L.roomrate and $R$.roomrate are identified as synonyms.

To perform the regression analysis, we choose one of the synonyms as the dependent variable. Suppose L.roomrate is chosen for this purpose, with taxrate and $R$.roomrate as independent variables. Since we have two independent variables, a multiple regression model is used. Let $Y$ denote L.roomrate, $x_{1}$ and $x_{2}$ denote taxrate and $R$.roomrate respectively. The regression analysis is to find the coefficients, $\beta_{i}, 1 \leq i \leq 3$ of the following model:

$Y=\beta_{1} x_{1}+\beta_{2} x_{2}+\beta_{3} x_{1} x_{2}+\epsilon$

By applying the multiple regression analysis to the given dataset, we obtain the following result: 


$$
\begin{aligned}
& \beta_{1}=-4.61989 \mathrm{E}-14 \\
& \beta_{2}=1.000000 \\
& \beta_{3}=0.010000 \\
& \epsilon=0.000000
\end{aligned}
$$

Since $\beta_{1}$ is negligible, we obtain the following by substituting the variables with the original attribute names:

$$
\text { L.roomrate }=\text { R.roomrate }+0.01 * \text { taxrate } * \text { R.roomrate }
$$

The preceding result can be rewritten into the following Horn clause

$$
\begin{aligned}
& \text { L.hotel(hcode, state, breakfast, facilities, roomrate } 2) \leftarrow \\
& \text { R.tax(state, taxrate }), \\
& \text { R.hotel(hcode, state, breakfast, facilities, roomrate1 }), \\
& \text { roomrate } 2=\left(1+0.01{ }^{*} \text { taxrate }\right){ }^{*} \text { roomrate1. }
\end{aligned}
$$

which is what we have used in creating the synthetic databases for this experiment.

\subsection{Experiment Two}

In this experiment, we modified the test data set by deleting the attribute taxrate. In other words, we have a data set of 1,000 tuples, comprising of the attributes: (hcode, state, L.roomrate, breakfast, facility, R.roomrate).

\section{Identifying semantically-related attributes \& define synonyms}

Similar to what was described in the previous experiment, we concluded from the partial correlation analysis that L.roomrate is highly correlated to R.roomrate. As before, the user may choose to treat L.roomrate and $R$.roomrate as synonyms. This prompts us to proceed with the next step.

\section{Discovering the relationships}

Applying the regression analysis to the data set, we obtained the following:

L.roomrate $=1.07 *$ R.roomrate -0.88

where the coefficient of determination $R^{2}=0.99123$. 


\section{Partitioning the data set}

Since $R^{2}$ is not equal to one, we may suspect that the equation obtained does not represent the true relationship among the attributes. (A highvalue for $R^{2}$ may however be acceptable if the values are known to be "noisy".) One approach for circumventing this problem is to partition the data set so that a more "consistent" relationship can be identified. For this purpose, we note that there are three categorical attributes: state, breakfast, and facility, with 40,2, and 3 distinct values respectively. Consequently, we partition the dataset according to each of the three attributes in turn and regression analysis was applied to each partition. The results can be summarized as follows:

\section{- Partitioning based on state}

Since there are 40 distinct values in the dataset, 1000 tuples were partitioned into 40 partitions. For each partition, regression analysis was applied. We obtained the following results:

\begin{tabular}{lll}
\hline State & Equation discovered & $R^{2}$ \\
\hline 0 & L.roomrate $=1.00 *$ R.roomrate & 1 \\
1 & L.roomrate $=1.09 *$ R.roomrate & 1 \\
& $\cdots$ & \\
38 & L.roomrate $=1.08 *$ R.roomrate & 1 \\
49 & L.roomrate $=1.04 *$ R.roomrate & 1 \\
\hline
\end{tabular}

- Partitioning based on breakfast

There are 2 distinct values for breakfast in the data set, 1000 tuples were partitioned into 2 partitions. The result of regression analysis is as follows:

\begin{tabular}{lll}
\hline Breakfast & Equation discovered & $R^{2}$ \\
\hline 1 & L.roomrate $=1.06^{*}$ R.roomrate -0.133591 & 0.99189 \\
2 & L.roomrate $=1.07^{*}$ R.roomrate -1.724193 & 0.99121 \\
\hline
\end{tabular}

- Partitioning based on facility

There are 3 distinct values for facility in the data set, 1000 tuples were partitioned into 3 partitions. The result of regression analysis is as follows: 


\begin{tabular}{lll}
\hline Facility & Equation discovered & $R^{2}$ \\
\hline 1 & L.roomrate $=1.066^{*}$ R.roomrate -1.702022 & 0.99235 \\
2 & L.roomrate $=1.058^{*}$ R.roomrate +0.708756 & 0.99187 \\
3 & L.roomrate $=1.076^{*}$ R.roomrate -1.728803 & 0.99070 \\
\hline
\end{tabular}

It is obvious that state is the best choice for achieving a partitioning that yields a consistent relationship.

From the equation that is discovered, we can create a new relation, called ratio, which reports the tax-rate for each of the $\mathbf{4 0}$ states:

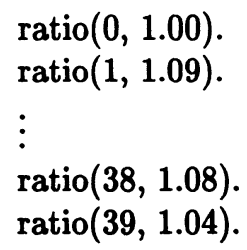

This in turn allows us to write the conversion rules:

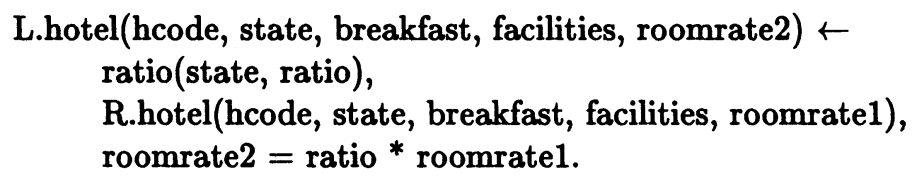

\subsection{Experiment Three}

In this final experiment, we created two synthetic relations which are presumably obtained from two distinct sources:

stock (scode, currency, volume, high, low, close);

stk_rpt (scode, price, volume, value);

Relation stk_rpt is derived from the source relation stock. For this experiment, we generated 500 tuples. The domain of the attributes are listed in the following table, together with their variable names used in the analysis. 


\begin{tabular}{llll}
\hline Variable & Relation & Attribute & Value Range \\
\hline X1 & stock, stk_rpt & scode & {$[1,500]$} \\
X2 & stk_rpt & price & stock.close*exchange_rate[stock.currency] \\
X3 & stk_rpt & volume & stock.volume ${ }^{*} 1000$ \\
X4 & stk_rpt & value & stk_rpt.price ${ }^{*}$ stk_rpt.volume \\
X5 & stock & currency & $1,2,3,4,5$ \\
X6 & stock & volume & $20-500$ \\
X7 & stock & high & {$\left[\right.$ stock.close, $1.2^{*}$ stock.close] } \\
X8 & stock & low & {$[0.85 *$ stock.close, stock.close] } \\
X9 & stock & close & {$[0.50,100]$} \\
\hline
\end{tabular}

It can be seen that, there are rather complex relationship among the attributes. We proceed with conflict detection and reconciliation as before.

\section{Identifying correlated attributes}

The zero-order partial correlation analysis on the data set gave the result as shown below:

\begin{tabular}{rrrrrrrrr} 
& $\mathrm{X} 1$ & $\mathrm{X} 2$ & $\mathrm{X} 3$ & $\mathrm{X} 4$ & $\mathrm{X} 5$ & $\mathrm{X} 6$ & $\mathrm{X} 7$ & $\mathrm{X} 8$ \\
\hline $\mathrm{X} 1$ & 1.0000 & & & & & & & \\
$\mathrm{X} 2$ & .0508 & 1.0000 & & & & & & \\
$\mathrm{X3}$ & -.0829 & -.0607 & 1.0000 & & & & & \\
$\mathrm{X} 4$ & .0425 & .7968 & .3243 & 1.0000 & & & & \\
$\mathrm{X} 5$ & -.0311 & .6571 & -.0645 & .5416 & 1.0000 & & & \\
$\mathrm{X6}$ & -.0829 & -.0607 & 1.0000 & .3243 & -.0645 & 1.0000 & & \\
$\mathrm{X} 7$ & .0762 & .4277 & -.0307 & .3331 & -.0245 & -.0307 & 1.0000 & \\
$\mathrm{X} 8$ & .0661 & .4355 & -.0309 & .3318 & -.0217 & -.0309 & .9905 & 1.0000 \\
$\mathrm{X} 9$ & .0725 & .4397 & -.0349 & .3357 & -.0193 & -.0349 & .9946 & .9959 \\
\hline
\end{tabular}

Using 0.1 as the threshold value and treating variables representing attributes from stk_rpt as the dependent variables, we identify the following correlated variable sets:

Table 5 Correlated Attributes Sets

\begin{tabular}{ccl}
\hline No & Dependent Variable & Independent Variables \\
\hline 0 & $\mathrm{X} 3$ & $\mathrm{X} 6$ \\
\hline 1 & $\mathrm{X} 2$ & $\mathrm{X} 4, \mathrm{X} 5, \mathrm{X} 7, \mathrm{X} 8, \mathrm{X} 9$ \\
\hline 2 & $\mathrm{X} 3$ & $\mathrm{X} 4$ \\
\hline 3 & $\mathrm{X} 4$ & $\mathrm{X} 2, \mathrm{X} 3, \mathrm{X} 5, \mathrm{X} 7, \mathrm{X} 8, \mathrm{X} 9$ \\
\hline
\end{tabular}




\section{Discovering relationships among related attributes}

Each correlated attribute set was analyzed using regression analysis to discover the relationships.

- Set 0:

Since the coefficient of $X 3$ and $X 6$ is 1 and the values of $X 3$ are not equal to that of $X 6$, linear regression analysis was conducted on these two variables. The following result was obtained:

$$
X 3=1000 * X 6
$$

This is consistent with the semantics of data used in creating the synthetic databases.

Set 1:

A brute force method is to use the following model which considers all of the one and two variable terms:

$$
\begin{aligned}
X 2 & =B 1 * X 4+B 2 * X 5+B 3 * X 7 \\
& +B 4 * X 8+B 5 * X 9 \\
& +B 6 * X 4 * X 5+B 7 * X 4 * X 7 \\
& +B 8 * X 4 * X 8+B 9 * X 4 * X 9 \\
& +B 10 * X 5 * X 7+B 11 * X 5 * X 8 \\
& +B 12 * X 5 * X 9
\end{aligned}
$$

The following regression equation was obtained with $R^{2}=0.90602$ :

$$
\begin{aligned}
X 2 & =1.2762 * X 5+1.6498 * X 7+1.0075 * X 8 \\
- & 2.0270 * X 9-2.4475 * X 5 * X 7 \\
- & 0.5275 * X 5 * X 8+3.8573 * X 5 * X 9
\end{aligned}
$$

Since the the coefficient of determination $R^{2} \neq 1$, we partitioned the data according to the only categorical variable $X 5$. Since $X 5$ has five distinct values, the 500 tuples were partitioned into 5 partitions. Furthermore, the result of model 5 reveals that coefficients $\mathrm{B} 1, \mathrm{~B} 6, \mathrm{~B} 7, \mathrm{~B} 8$, and $\mathrm{B} 9$ are zero. We can construct for each partition a simpler model

$X 2=B 3 * X 7+B 4 * X 8+B 5 * X 9$ 
The regression results are given below:

\begin{tabular}{lll}
\hline $\mathrm{X5}$ & equation discovered & $R^{2}$ \\
\hline 0 & $\mathrm{X} 2=0.4^{*} \mathrm{X} 9$ & 1 \\
1 & $\mathrm{X} 2=0.7^{*} \mathrm{X} 9$ & 1 \\
2 & $\mathrm{X} 2=1.0^{*} \mathrm{X} 9$ & 1 \\
3 & $\mathrm{X} 2=1.8^{*} \mathrm{X} 9$ & 1 \\
4 & $\mathrm{X} 2=5.8^{*} \mathrm{X} 9$ & 1 \\
\hline
\end{tabular}

In fact, the coefficients shown in the above table are the exchange rates used in generating stk_rpt.price from stock.close based on stock.currency. The regression analysis correctly discovered this fact automatically.

\section{Set 2:}

As the $R^{2}$ value of the regression analysis on the whole data set is not equal to 1 , we repeat the regression analysis after partitioning the data set based on $X 5$. The results are as follows:

\begin{tabular}{lcccl}
\hline Data Set & X5 & Equation Discovered & $R^{2}$ \\
\hline nonpartitioned & all & $\mathrm{X} 3=0.0012^{*} \mathrm{X} 4+232938.0964$ & 0.10517 \\
\hline partition & 0 & $\mathrm{X} 3=0.2005^{*} \mathrm{X} 4+163787.2507$ & 0.37291 \\
& 1 & $\mathrm{X} 3=0.1259^{*} \mathrm{X} 4+154285.2230$ & 0.41848 \\
& 2 & $\mathrm{X} 3=0.0068^{*} \mathrm{X} 4+180850.1938$ & 0.38318 \\
& 3 & $\mathrm{X} 3=0.0054^{*} \mathrm{X} 4+144068.7345$ & 0.35892 \\
& 4 & $\mathrm{X} 3=0.0016^{*} \mathrm{X} 4+126240.6993$ & 0.50320 \\
\hline
\end{tabular}

In both instances, no consistent relationships were found.

\section{- Set 3:}

The regression analysis on set 4 yields the following result:

$$
X 4=X 2 * X 3
$$

$$
\text { with } R^{2}=1 \text {. }
$$

The above results can now be summarized in the following conversion rule: 


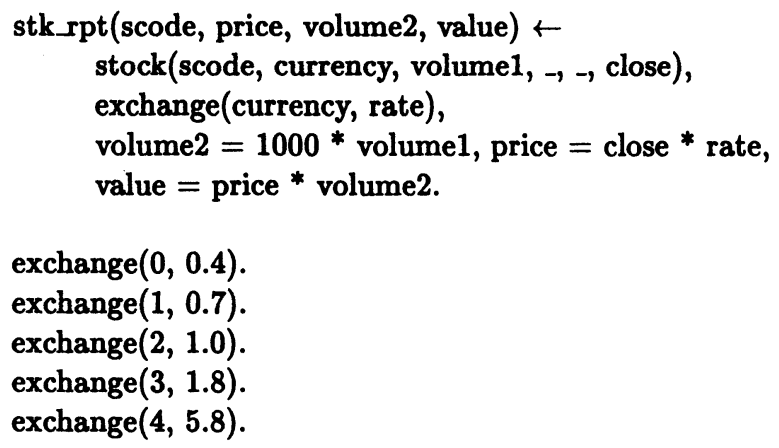

where exchange is a new relation with two attributes (currency, rate), in which rate is presumably the exchange-rate for converting other currencies into the currency used in stk_rpt.

\section{CONCLUSION}

The quest for semantic interoperability among autonomous and heterogeneous information systems has led to a proliferation of prototypes. However, as far as we know, little work has been done on the automatic detection and discovery of semantic conflicts. In this paper, we have presented the motivation for this problem, and have demonstrated how conflict detection and resolution can be mostly automated using various statistical techniques.

We also presented three experiments in which the techniques were applied to uncover hidden relationships between data elements. While it is clear that we have only addressed a small subset of the problems, we are convinced that the work reported here remains significant for the role it plays in breaking the ground for an important class of problems.

We presented in this paper only a generic approach towards data integration using statistical techniques. A number of issues have not been well addressed. For example, we have been only dealing with the quantitative relationship among numerical attributes. There are other statistical techniques available to analyze ordinal or categorical data. It is one of our future work to include those techniques into the system so that conversion functions involving categorical data can be discovered. Similarly, regression is only effective for discovering linear relationships. Other techniques, such as neural networks need to be explored to discover non-linear relationships.

Furthermore, all example data used in this paper are synthetic without noises. In real world, data are usually contaminated with errors caused by various reasons. Although we argue that with clean data, $R^{2}=1$ can be used as the criterion of accepting the conversion functions discovered, it may not be so if the data contain noises. Our on-going work has indicated that, combining outlier detection with regression techniques, we can not only discover conversion functions with noise data, but also find the tuples containing erroneous values. It is an interesting work to extend the basic approaches to address the data cleansing problem.

The astute reader may also object to the approach proposed in this paper by pointing out that the statistical techniques are expensive and cannot be used to 
support ad hoc queries at real-time. Our response is that it is never our intention to apply the regression methods at real-time. On the other hand, data analysis should be performed off-line and at the time when a new system is brought into the "federation" of inter-operating systems. The rules which are discovered during this process can be used as input to the definition of a shared schema where the conflicts are being resolved. In loosely-coupled systems where shared schemas are not defined a priori, the rules may be stored as part of a user's profile which can be drawn upon (as part of the local knowledge base) whenever a query is to be formulated.

We are currently also examining extensions to this work that will allow it to be incorporated into a Context Interchange System (Bressan, Fynn, Goh, Jakobisiak, Hussein, Kon, Lee, Madnick, Pena, Qu, Shum \& Siegel 1997). The key observation here is that the extraction of conversion functions alone is not sufficient, and that further work is needed to be able to also uncover the meta-data that is associated with different information sources. For example, in the case of Experiment 2, it is not good enough to identify the ratios (state-tax) that are needed for computing the tax-value: it would have been more valuable if we are able to match the ratios to yet another data source furnishing the state-tax percentage and figure out instead that different hotels have different tax rates by virtue of its location. Thus, if we know indeed that all hotels reported in a given database have a uniform tax rate of $6 \%$, we might formulate a hypothesis that these are all hotels located in Massachusetts. In principle, these conclusions can be derived using the same statistical techniques as those which we have described. Further work however remains necessary to verify their efficiency.

\section{REFERENCES}

Bressan, S., Fynn, K., Goh, C., Jakobisiak, M., Hussein, K., Kon, H., Lee, T., Madnick, S., Pena, T., Qu, J., Shum, A. \& Siegel, M. (1997), The COntext INterchange mediator prototype, in 'Proc. ACM SIGMOD/PODS Joint Conference', Tuczon, AZ. To appear.

Falkenhainer, B. \& Michalski, R. (1986), 'Integrating quantitative and qualitative discovery: The abacus system', Machine Learning 4(1), 367-401.

Kokar, M. (1986), 'Determining arguments of invariant functional description', $M a$ chine Learning 4(1), 403-422.

Langley, P., Simon, H., G.Bradshaw \& Żytkow, M. (1987), Scientific Discovery: An Account of the Creative Processes, MIT Press.

Scheuermann, P., Yu, C., Elmagarmid, A., Garcia-Molina, H., Manola, F., McLeod, D., Rosenthal, A. \& Templeton, M. (Dec 1990), 'Report on the workshop on heterogeneous database systems', ACM SIGMOD RECORD 4(19), 2331. Held at Northwestern University, Evanston, Ilinois, Dec 11-13, 1989. Sponsored by NSF.

Sheth, A. \& Larson, J. (1990), 'Federated database systems for managing distributed, heterogeneous, and autonomous databases', ACM Computing Surveys 3(22), 183-236.

Wu, Y. \& Wang, S. (1991), Discovering functional relationships from observational data, in 'Knowledge Discovery in Databases', The AAAI Press.

Żytkow, J. \& Baker, J. (1991), Interactive mining of regularities in databases, in 'Knowledge Discovery in Databases', The AAAI Press. 


\section{BIOGRAPHY}

Hongjun Lu, currently a senior lecturer in the National University of Singapore, received his $\mathrm{PhD}$ degree from the University of Wisconsin, Madison. His research interests include query processing and optimization, parallel and distributed database systems, and knowledge discovery and data mining. He has published more than $\mathbf{8 0}$ papers in various database conferences and journals. He is currently a member of the ACM SIGMOD Advisory Committee and has served as a programme committee member for many leading database conferences and as a reviewer for major database journals and conferences.

Weiguo Fan received the B.E. degree in Information Science and Engineering from Xi'an Jiaotong University, Xi'an, P.R. China, in 1995 and M.S. degree in Computer Science from the National University of Singapore, Singapore, in 1997. Since then, he has been working as a research assistant on the project of Establishing Context for Information Integration Using Data Mining Techniques with Dr. Hongjun Lu. His research interests include data mining, database integration, data quality, electronic commerce and operations research.

Cheng Hian Goh received his $\mathrm{PhD}$ from MIT and has since returned to be a faculty member at the National University of Singapore. He has published in different areas including relational database theory, conceptual data modeling, logic programming, information integration, and database query optimization. His current research interests include information retrieval on the WWW, and the changing economic landscape brought about by the network economy.

Stuart E. Madnick is the John Norris Maguire Professor of Information Technology and Leaders for Manufacturing Professor of Management Science at the MIT Sloan School of Management. He is also an affiliate member of the MIT Laboratory for Computer Science and a member of the Executive Committee of the MIT Center for Information Systems Research. His current research interests include connectivity among disparate distributed information systems, database technology, and software project management. He is the author or co-author of over 200 books, articles, or reports on these subjects, including the classic textbook, Operating Systems (McGraw-Hill), and the book, The Dynamics of Software Development (PrenticeHall). He has been active in industry, making significant contributions as one of the key designers and developers of projects such as IBM's VM/370 operating system and Lockheed's DIALOG information retrieval system.

David W. Cheung received the $\mathrm{MSc}$ and $\mathrm{PhD}$ degree in computer science from Simon Fraser University, Canada, in 1985 and 1989, respectively. He also received the BSc degree in mathematics from the Chinese University of Hong Kong. From 1989 to 1993, he was with Bell Northern Research, Canada, where he was a member of the scientific staff. Currently, he is an associate professor of the Department of Computer Science at the University of Hong Kong. His research interests include distributed databases, spatial databases, data mining and data warehousing. 